\title{
Real-time dosimeter targeted to nuclear applications
}

\author{
Alexandre Correia $^{1 *}$, Carla C. Rosa ${ }^{1,2}$, Pedro M. P. Santos ${ }^{3}$, António N. Falcão ${ }^{3}$, Katharina Loren $\uplus^{3}$ \\ ${ }^{1}$ INESC TEC, Optoelectronics and Electronics Systems Unit, Porto, Portugal \\ ${ }^{2}$ Department of Physics and Astronomy, Faculty of Sciences (FCUP), University of Porto, Portugal \\ ${ }^{3}$ Instituto Superior Técnico (IST), Campus Tecnológico e Nuclear (CTN), Laboratory of \\ Accelerators and Radiation Technologies, Estrada Nacional 10, Bobadela LRS, Portugal
}

\begin{abstract}
An intrinsic fiber optic dosimeter (FOD) targeted to nuclear applications is presented. The proposed real-time dosimeter provides dose information based on the historic record over time of the effects of ionizing radiation on single- and multimode pure silica fibers, and also on PMMA plastic fibers. The effect of ${ }^{60} \mathrm{Co}$ gamma irradiation on optical links based on silica and plastic fibers were assessed, considering thermal environment effects over a wide range of variation of the operating parameters. Cerenkov radiation and radiation-induced absorption effects were in focus. The corresponding distortion and spectral transmission degradation were evaluated over wide range of the operating parameters. Radiation induced attenuation (RIA) has shown a spectral band dependent behaviour up to $840 \mathrm{~Gy}$ dose levels. The performance of different fibers was assessed against the performance of non-irradiated fibers. From the measurements of dose rate and total dose imparted by ionizing radiation in the fibers we verified that fibers with radiation resistance issues showed wavelength-dependent radiation sensitivity increasing with dose rate.

Upon evaluation of correlations between the total dose, the induced loss at various dose rates and different wavelengths, it was concluded that intrinsic fiber dosimeters can be used for dose rates in the range 4 - $28 \mathrm{~Gy} / \mathrm{min}$., typical of severe radiation environments.
\end{abstract}

Keywords: optical fibers, radiation induced absorption, dosimetry and intrinsic sensors.

\section{INTRODUCTION}

Real-time dosimetry is being pushed towards meeting the increasing requirement for in situ dosimeters for oncologic treatments in order to assess the correspondence between treatment planning and dose delivery, as well as, the need to monitor high doses of ionizing radiation in industrial applications. The need for real-time measurements is becoming more important and researchers are continually looking for ways to improve dosimetric systems, increasing the sensitivity and reducing costs [1], and to design probes with an optimized housing, transducer stability and biocompatibility [2, 3]. Due to many advantages, namely the easy integration into a wide variety of structures, the high resistance to severe radiation environments, their small size and their immunity to electromagnetic and chemical interference, as well as their and remote sensing capabilities, fiber optic dosimeters (FOD) are good candidates to be operated as stand-alone devices, avoiding handling problems, but also ensuring safe operation conditions in ionizing radiation environments [3,4]. Proved to be essential in several application fields, such as clinical applications, sterilization of products and food industry, among others, the success of fiber optics applications are linked to their excellent linearity in high temperature environments, adequate mass absorption coefficients and tissue-equivalent characteristics, among others $[5,6]$. On the other hand, the adequacy of optical fibers to act as intrinsic sensors for monitoring high doses of gamma radiation for industrial and nuclear applications is well known, as well as their use for real-time motorization of low doses of ionizing radiation, such as x-ray and gamma rays [7]. The FOD should have a small sensitive volume combined with thin, flexible, robust and lightweight properties to make them promising devices to be used in different and complex environments taking advantage of their ability to monitor sites far away from the controller [5, 8].

Researchers showed [7, 9] that, at the microscopic scale, radiation generates point defects in the amorphous silica glass network (a-SiO2) through ionization or "knock-on" processes. These point defects, or color centers, induce the appearance of new energy levels located inside the band gap of the dielectric. As a consequence, at the macroscopic

\footnotetext{
*Corresponding author: amrc@inescporto.pt
} 
scale, the defect-containing glass absorbs a more important part of transmitted light signals, leading to an increase of the attenuation of the fiber. Studies have been focused on the radiation effect on optical fibers and associated defect center generation, aiming at using the fiber optic as sensor under severe radiation environments, such as nuclear waste tanks, nuclear reactors, among others [3,9]. The energy losses associated with the interaction between highly energetic radiation and matter, can imply atoms ionization or can induce non-ionizing effects, such as changes of the vibrational/rotational states of molecules, atoms vibrations or atoms displacement. When exposing optical fibers to ionizing radiation, structural changes are induced in the material, leading to radiation induced absorption (RIA) that depends largely on core clad composition, dopant concentration, fiber design, along with different radiation parameters like radiation source, dose rate and operating parameters, such as temperature and wavelength [4].

A Cerenkov radiation is also generated by irradiation of the optical fiber, which constitutes a common and unwanted background signal, usually referred to as stem effect, by the formation of energetic charged particles that pass through a medium with a velocity greater than that of light [10]. The radiation from Cobalt source $\left({ }^{60} \mathrm{Co}\right)$ is able to produce secondary electrons in irradiated materials with sufficiently high energies for Cerenkov radiation production in fiber optics, within a peak spectral range between 400 to $480 \mathrm{~nm}$ [10]. This has also to be considered in the selection of prospective candidates for real-time radiation dosimetry.

In this paper we report results of a preliminary study of the effect of ionizing radiation on the optical transmission of specific optical fibers, aiming at evaluating their use as real-time dosimeters when placed in high energy radiation fields.

\section{REAL-TIME FIBER OPTIC DOSIMETER}

The radiation induced structural changes affect the optical fiber propagation characteristics and; the FOD allows direct and quasi-simultaneous reading of the absorbed doses. Moreover, the signal measurement can be multiplexed allowing the mapping of the dose imparted at different field locations. Due to the application aimed at, ideally, the fibers should have, after irradiation, an almost complete recovery of their optical properties. This work addresses the response of common optical fibers, and assesses stem effect minimization. The fibers that have been tested are mainly made of pure silica and PMMA. More detailed information is given in the next section.

\subsection{Set-up of real-time dosimeter}

Figure 1 shows the schematic diagram used to measure the optical signal transmission under a severe radiation environment. Sample irradiation was performed at the PRECISA 22 irradiation facility installed at the Campus Tecnológico e Nuclear, of the Instituto Superior Técnico (IST), Loures, Portugal. PRECISA 22 is equipped with a ${ }^{60}$ Co gamma source, which emits gamma rays with characteristic energies of 1.17 and $1.33 \mathrm{MeV}$. Four sources with different activities are available.

The sensing probes, $50 \mathrm{~cm}$ long each, were coiled into circles with a diameter of $10 \mathrm{~mm}$, and placed inside the ${ }^{60} \mathrm{Co}$ chamber in specific irradiation positions to which correspond different dose rates. The irradiated coils are connected to external extensions - one connected to a remote light measuring device, and the other to a tungsten halogen light source (HL-2000, Ocean Optics). The tested sensing probes materials were:

- Pure silica $\left(\mathrm{SiO}_{2}\right)$

○ Multimode core diameter of $105 \mu \mathrm{m}$, a cladding doped with fluorine (AFS105-125, Thorlabs),

- Single mode with mode field diameter of $4 \mu \mathrm{m}$ at $630 \mathrm{~nm}$, with a acrylate buffer coating (FS-SN3221, 3M)

- PMMA (poly-methyl-methacrylate, $\mathrm{C}_{5} \mathrm{H}_{8} \mathrm{O}_{2}$ ), $1000 \mu$ m diameter, the cladding is a fluorinated polymer with a black polyethylene buffer (RS 368-053, RS Electronics).

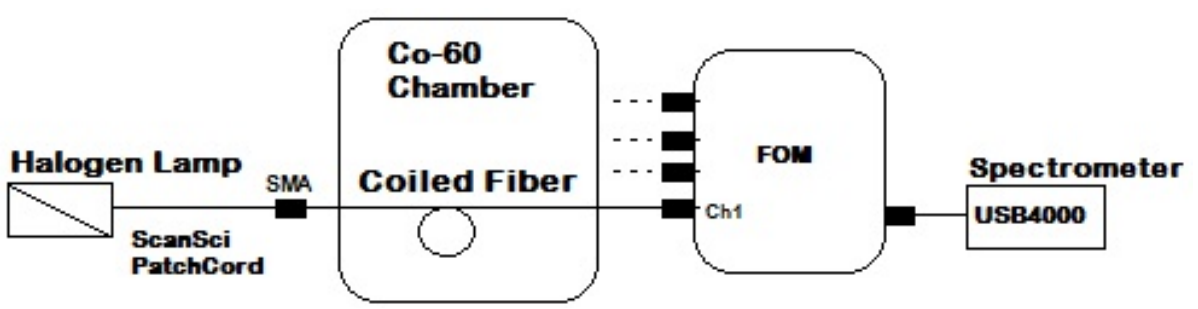

Figure 1 - Schematic diagram of the experimental arrangement. 


\subsection{Signal acquisition}

Dose rates at the irradiation positions were determined using a calibrated ionization chamber. To evaluate the Cerenkov radiation, which depends on the type of optical fiber, its thickness, the angle between the gamma-ray trajectory and the optical fiber axis and the energy of the gamma-ray, among others, for each test sensing probe irradiated, an additional whole bare fiber was also prepared and irradiated in the same conditions as those of the test sensing probe, but measured without injecting the signal from the HL-2000.

Outside the radiation bunker, The SMA terminated FOD probes were linked to another low -OH multimode silica fiber (BFL-48, ScanSci patch-cord) with $600 \mu \mathrm{m}$ diameter and $3 \mathrm{~m}$ length, either directly through an SMA-SMA connector, or interfaced with a Fiber Optic Multiplexer (8x2 channels, Avantes). An index matching gel (Thorlabs) was used to maximize the optical coupling of the test probe to the transmitting patch-cord (SMA-SMA connector).

Real-time motorization was provided by an optical Spectrometer (USB4000, Ocean Optics) operating in the range 350 $1000 \mathrm{~nm}$, from which we obtained the optical fiber transmission spectra acquired in time, during irradiation. The transmitted signal was also measured in the infrared range of $900-1700 \mathrm{~nm}$, using a NIR spectrometer (NIRQuest 512, Ocean Optics). The Cerenkov radiation was measured using different detectors (femtowatt PDF10A, PDF10C - Thorlabs and MPPC-128 - Hamamatsu). The femtowatt photo-receivers combine ultra-low noise Si photodiode with specially designed trans-impedance amplifiers, offering extremely high gain. The MPPC, Multi-Pixel Photon Counter contains a thermoelectrically cooled device capable of detecting ultra-low light, in the visible spectral window 320 - $900 \mathrm{~nm}$. The electrical signal was acquired with an acquisition card (DAQ USB-6351, National Instruments). Control and signal processing were performed with the aid of Labview software (National Instruments).

\subsection{Radiation exposure procedure}

The Radiation-Induced Attenuation (RIA) strongly varies with the kind of fiber used. As observed, the attenuation decreases with increasing injected power or decreasing dose-rate and has a strong dependence on temperature. The intrinsic FOD is directly affected by radiation-induced absorption effect, which degrades the transmitted signal, as in some spectral bands the attenuation becomes radiation dependent, up to $220 \mathrm{~Gy}$. The Precisa- 22 has four ${ }^{60}$ Co sources controlled with a remote afterload; allowing irradiation dose rates between $0.01 \mathrm{~Gy} / \mathrm{min}$. and $30 \mathrm{~Gy} / \mathrm{min}$., depending on the e sample holder placement inside the irradiation chamber and the number of ${ }^{60} \mathrm{Co}$ sources selected. The sample container was a plastic cylindrical box. Table 1 shows the irradiations conditions used. The irradiation time was set to thirty minutes at a specific dose rate. After irradiation, the fiber optic transmission recovery was also analyzed.

\begin{tabular}{|c|c|c|c|c|}
\hline \multicolumn{2}{|c|}{$\begin{array}{c}\text { irradiation conditions } \\
\text { FOD } \\
\end{array}$} & $\begin{array}{l}\text { dose rate } \\
{[\mathrm{Gy} / \mathrm{min} .]}\end{array}$ & $\begin{array}{l}\text { total dose } \\
{[\text { Gy] }}\end{array}$ & $\begin{array}{l}\text { irradiation time } \& \\
\text { recovery time [min.] }\end{array}$ \\
\hline $\mathrm{MM}^{-\mathrm{SiO}_{2}}$ & \multirow{3}{*}{$\begin{array}{c}50 \mathrm{~cm} \text { length } \\
\text { (coiled into } 1 \mathrm{~cm} \text { ) }\end{array}$} & 4.0 to 11.3 & 320 & \multirow{3}{*}{$30 / 30$} \\
\hline $\mathrm{SM}^{-\mathrm{SiO}_{2}}$ & & 4.0 to 11.3 & & \\
\hline PMMA & & 4.0 to 28.0 & 840 & \\
\hline
\end{tabular}

Table 1 - Irradiation conditions applied to optical fibers.

\subsection{RIA Measurements}

RIA measurements were performed in the VIS range 500-900 nm and in NIR range 900-1700 nm. The sample exposures were performed at different dose rates, in the interval $4.0-28-0 \mathrm{~Gy} / \mathrm{min}$. The temperature was monitored in each stage. At an early exposure stage, lower dose rates of $1.0 \mathrm{~Gy} / \mathrm{min}$. were used to allow the collection of data in conditions similar to those of typical radiotherapy exposure. The transmission spectra were monitored in situ and were recorded every ten seconds.

\section{RESULTS AND DISCUSSION}

Visible light transmission spectra obtained with the multimode silica fiber are shown in figure 2. For lower dose rates of $1.0 \mathrm{~Gy} / \mathrm{min}$. no change in absorption is observed. However, for higher dose rates it appears that the RIA slowly increases saturating after a certain irradiation time, above which the transmission spectra remain unchanged.

Fig. $2 \mathrm{~b}$ shows the recovery of the fiber transmission for different wavelength regions as a function of the time passed after the radiation stopped. The transmission intensities were normalized to those of the non-irradiated fiber. The attenuation in fiber transmission is clear for low wavelengths, notably around $500 \mathrm{~nm}$, and less marked, but still 
measurable, for higher wavelengths, with the exception of the near-infrared spectral window where little degradation was observed. The luminescence and Cerenkov light have very weak intensities along the fiber axis, and are only detectable by using the PDF10A detector (Thorlabs) that capture a background signal equal to $1.17 \mathrm{~V}$ during irradiation with the light source removed.

According to figure $2 \mathrm{~b}$, after thirty minutes of irradiation, the transmitted optical fiber signal degrades almost $80 \%$ at $500 \mathrm{~nm}$. However, after irradiation, the optical fiber starts recovering, reaching $56 \%$ of the reference fiber transmission (non-irradiated fiber) after 20 minutes. The recovery process can be accelerated by heating the irradiated fiber. As depicted from Fig. 2, the radiation sensitivity of the fiber is very high.
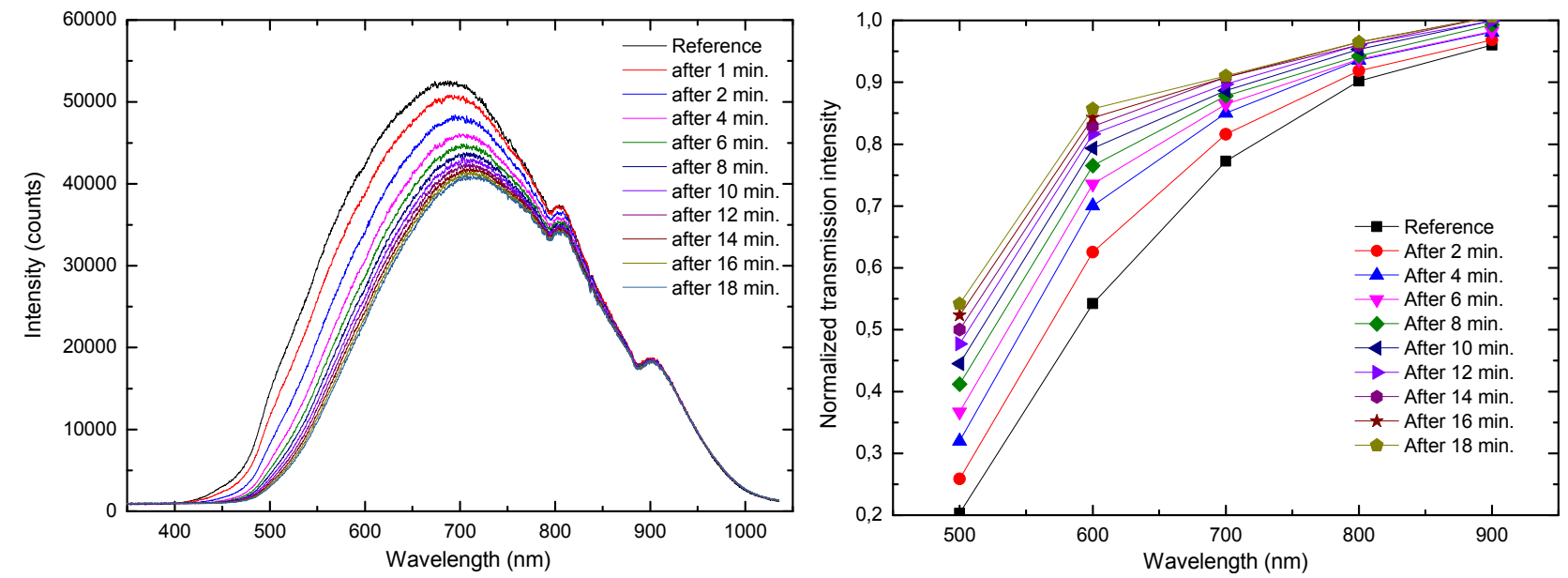

Figure 2 - Transmitted spectra of light originated by a HL-2000 light source after propagating along an optical fiber (AFS105-125): a) during irradiation at a dose rate of $11.3 \mathrm{~Gy} / \mathrm{min}$; b) transmission for different wavelengths during recovery phase, i.e after stopping the irradiation process - at $500 \mathrm{~nm}$ and after $30 \mathrm{~min}$. of irradiation, the optical transmission drops to $20 \%$ of its initial non-irradiated state.
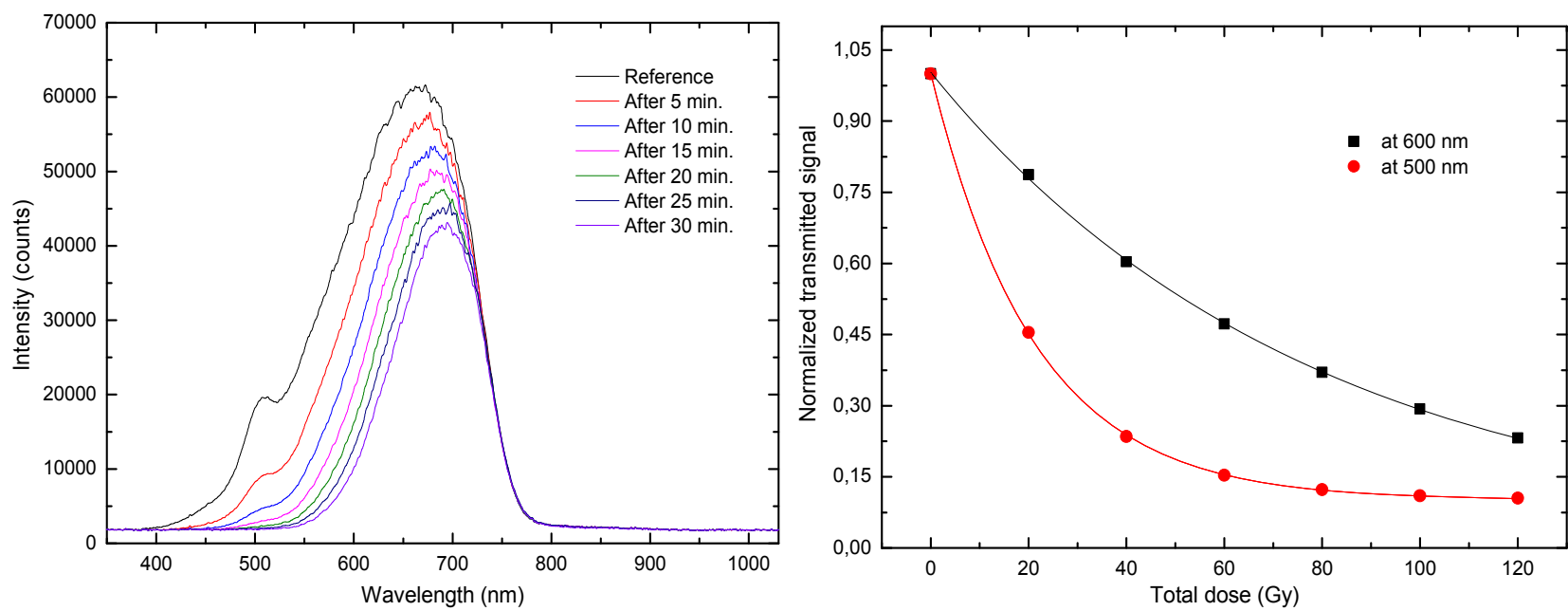

Figure 3 - Transmitted spectra of light originated by a HL-2000 light source after propagating along a single mode optical fiber (FS-SN-3221): a) during irradiation with a dose rate equal to $4 \mathrm{~Gy} / \mathrm{min}$; b) normalized transmitted signal evolution during ${ }^{60} \mathrm{Co}$ irradiation.

Figure 3, shows data obtained for optical fiber (FS-SN-3221), irradiated at room temperature in the range 350 to 1050 $\mathrm{nm}$. Similarly to what was observed for fiber (AFS105-125), the effect of irradiation on transmission is clear even at low irradiation doses. Again, the measurements provide evidence of a strong absorption in the UV, visible region and nearinfrared spectral window $(\lambda<800 \mathrm{~nm})$. The dynamics of these measurements should be improved in the future to provide a better characterization in this spectral range. Additionally, the single mode fiber has higher sensitivity for higher doses considering a peak spectrum of $600 \mathrm{~nm}$, and lower comparing with a wavelength of $500 \mathrm{~nm}$ (figure 3b). 
Figure 4 shows that the rate at which attenuation occurs is higher (i.e, making the probe more sensitive) at low doses, saturating for higher doses (i.e, the probe decreases sensitivity with increasing dose). The saturation is reached when the annealing effects and the radiation induced trapped charges balance each other so that the radiation induced attenuation reaches a constant value for increasing dose while at a constant dose rate.

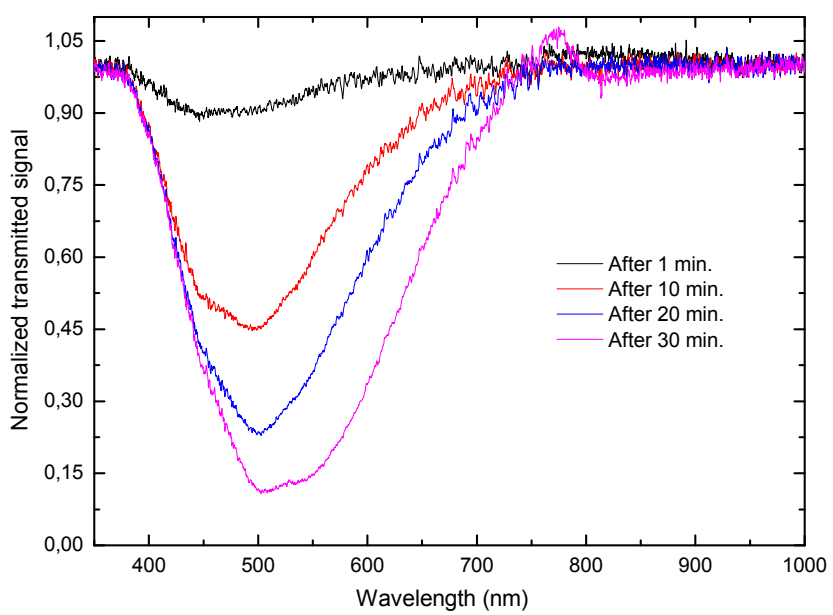

Figure 4 - Normalized transmitted spectra of light originated from a HL-2000 light source after propagating along a single mode optical fiber (FS-SN-3221), during a ${ }^{60} \mathrm{Co}$ irradiation at $4 \mathrm{~Gy} / \mathrm{min}$.
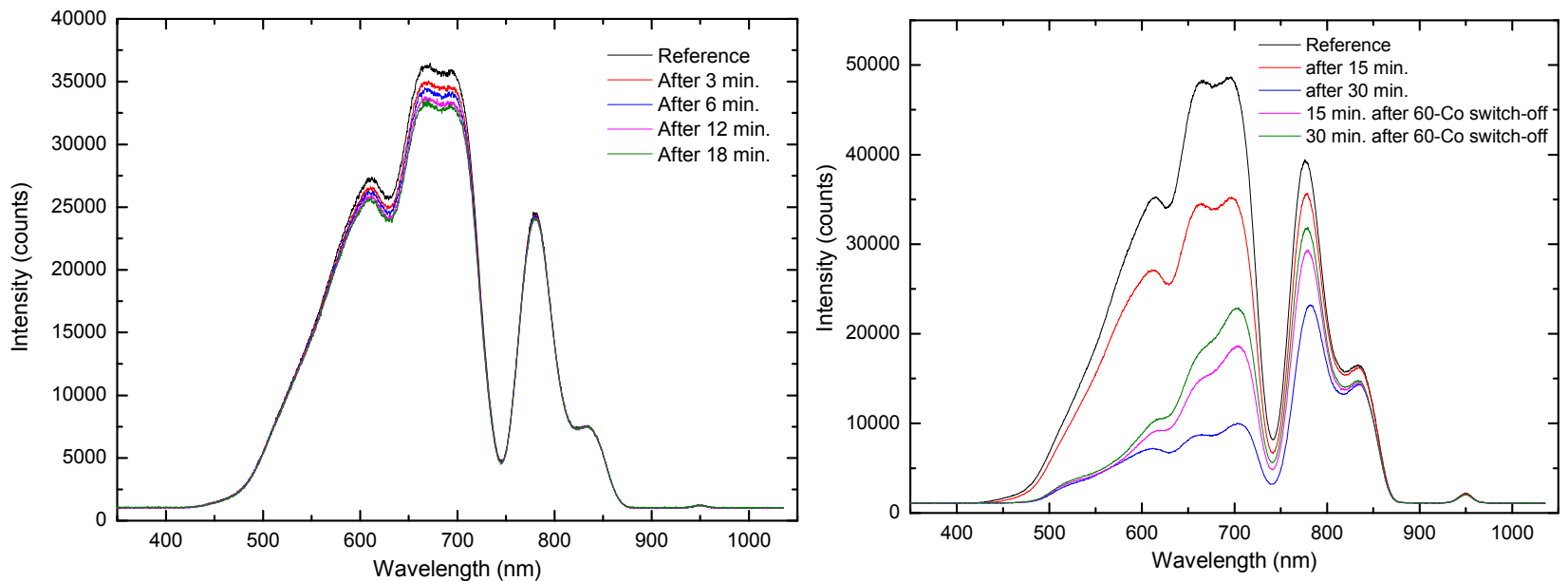

Figure 5 - Transmitted spectra of a HL-2000 light source by a PMMA plastic fiber (RS 413-374), during irradiation with: a) dose rate equal to $11,3 \mathrm{~Gy} / \mathrm{min}$ and $\mathrm{b}$ ) dose rate equal to $28,0 \mathrm{~Gy} / \mathrm{min}$.

In this preliminary study we also tested a plastic optical fiber, with a $1 \mathrm{~mm}$ diameter core. The sample was first irradiated and transmissions recorded as for the previously discussed fibers (Fig. 5a).

Then it was placed in an oven at $60^{\circ}$ Celsius for $12 \mathrm{~h}$. The transmission was then measured, and the sample irradiated once more at a higher irradiation dose rate (Fig. 5b). We observed that during the first irradiation, the loss in transmission was small for all wavelengths in the range $350 \mathrm{~nm}$ to $1050 \mathrm{~nm}$ (Figure 5a). After the 12 hour annealing process, the transmission had completely recovered - in fact it was higher than for the non-irradiated sample. However, and contrarily to what was observed in the first irradiation experiment, during the second irradiation, the optical attenuation was extremely marked (Figure 5b).

This effect has to be further investigated in order to clarify the effect of annealing and the effect of dose rate. The fibers exhibit good sensitivity, capable of monitoring doses up to $840 \mathrm{~Gy}$ for PMMA fibers, and in a range between 4 to 220 Gy for pure silica fibers. 


\section{CONCLUSION}

In conclusion, the read-out system for dosimetry with fiber optics combines the advantages of a direct reading, with a parallel, computer-controlled read-out, with high sensitivity and easy integration into a wide variety of structures, as a low cost solution. The luminescence and Cerenkov light are not observed in the experiments due to their weak intensities as compared to the light levels being coupled into the fiber.

The evaluation of the RIA process i.e. the degradation of the signal transmission of optical fibers upon irradiation was carried out by monitoring the signal in real time. The results demonstrate the feasibility of using optical fibers as an intrinsic sensor for in situ dose monitorization in the range of 4 to $220 \mathrm{~Gy}$ for silica fibers and up to $840 \mathrm{~Gy}$ for plastic fibers. For transmitted light at wavelengths greater than $900 \mathrm{~nm}$, absorption is hardly observed and the radiation induced loss tends to saturation with a rapid increase of total dose. After irradiation, recovery of the optical transmission occurs, and the same is suspected to occur during the irradiation process, with the loss of transmission resulting from the balance between the rates of local destruction of the fiber structure and recovery.

In conclusion, and even though further detailed studies need to be performed, it is clear that the results obtained are quite promising as to make use of the changes in the optical properties of optical fibers for real-time inexpensive and reliable dose monitoring in severe radiation environments, and clinical applications as well.

\section{ACKNOWLEDGEMENTS}

This work is financed by National Funds through the FCT - Fundação para a Ciência e Tecnologia, supporting the Sensing project with reference: NORTE-07-0124-FEDER-000058 and by the host institution INESC-Porto grant: AE2013-0120. The authors also thank the support of CTN, Laboratory of Accelerators and Radiation Technologies and Unit of Reactors and Nuclear Safety, (IST) Lisboa.

\section{REFERENCES}

[1] Jang, K., Cho, D., Yoo, W., Park, B., Moon, J., Kim, S., Cho, Y., “A Scintillating Fiber-optic Dosimeter for Co-60 Radiotherapy,” IEEE 978(1), 4244-5335 (2009);

[2] Becks, K. H,, Drees, J., Goldmann, K., Gregor, I. M., and Heintz, M., “A Multichannel Dosimeter Based on Scintillating Fibers for Medical Applications,” Nucl. Instrum. Phys. Res. A., 454, 147-151, (2000);

[3] Fuhua, L., Yuying A., Ping, W., Bibo, S., and Shaowu, C., "Effects of Radiation on Optical Fibers”, Recent Progress in Optical Fiber Research, Dr Moh. Yasin (Ed.), ISBN: 978-953-307-823-6, InTech, (2012);

[4] Sporea, D., Sporea, A., O’Keeffe, S., McCarthy, D. and Lewis, E.,’Optical Fibers and Optical Fiber Sensors Used in Radiation Monitoring,” Select Topics on Optical Fiber Technology, ISBN: 978-953-51-0091-1 (2012);

[5] D. McCarthy, S. O’Keeffe, G. Leen, E. Lewi, “Optical fibre radiation dosimetry for low dose applications”, Optical Fibre Sensors Research Centre, University of Limerick, (2012);

[6] Lecler, S., and Meyrueis, P., “Intrinsic Optical Fiber Sensor,” Fiber Optic Sensors, Dr Moh. Yasin (Ed.), ISBN: 978-953-307-9226, InTech, (2012);

[7] Regnier, E., Flammer, I., Girard, S., Gooijer, F., Achten, F., and Kuyt, G., "Low-Dose Radiation-Induced Attenuation at infraRed Wavelengths for P-Doped,Ge-Doped, Pure Silica-Core Optical Fibres,” IEEE Transactions on Nuclear Science, 54(4), (2007);

[8] Low, D. A., Moran, J. M., Dempsey, J. F., Dong, L., and Oldham, M., “Dosimetry tools and techniques for IMRT,” Med. Phys. 38, 3 (2011);

[9] O’Keeffe, S., Fitzpatrick, C., Lewis, E,. and Al-Shamma’a, A.I., “A review of optical fibre radiation dosimeters,” Sensor Review 28, 136-142 (2008);

[10] Law, S. H., Suchowerska, N., McKenzie, D. R., Fleming, S. C. and Lin, T., “Transmission of Cerenkov radiation in optical fibers,” Opt. Lett. 32, 1205-1207 (2007);

[11] Kim, K. A., Yoo, W. J., Jang, K. W., Moon, J., Han, K. T., Jeon, D., Park, J., Cha, E. J., and Lee, B., "Development of a fibreoptic dosemeter to measure the skin dose and percentage depth dose in the build-up region of therapeutic photon beams," Radiation Protection Dosimetry, 153 (3), 294-299 (2013); 